\title{
BAD BREATH IN GASTROINTESTINAL AND LIVER DISEASES
}

\author{
Radosveta Tomova ${ }^{1}$, Vladimir Panov ${ }^{2}$ \\ ${ }^{1}$ Clinic of Gastroenterology, University Hospital St "Ivan Rilski", Sofia \\ ${ }^{2}$ Department of Conservative Dentistry and Oral Pathology, Faculty of Dental Medicine, \\ Medical University of Varna
}

\begin{abstract}
Bad breath is an oral health condition characterized by unpleasant odor from the oral cavity. The origin of halitosis is associated with different oral pathologies, but it may be related to systemic conditions in $15 \%$ of the cases. We examined 55 patients with various hepatic and gastrointestinal diseases. With an apparatus to measure bad breath we investigated the levels of odor in patients. The apparatus quantifies volatile sulfur compounds, hydrogen sulfide, mercaptan, dimethyl sulfide and hydrocarbon from the mouth on a scale from 0 to 5 . We compared the obtained results with those of 79 patients with general good health. Our studies indicate that the levels of bad breath in the first group were significantly higher in men $(0.99)$ compared to those of women (0.41), more than two times, which is in contrast to the group of patients without systemic diseases. We registered the following average values for bad breath - 0.65 in younger patients (20-50 years) and a higher one - 0.89 in older patients (51-78 years). Older patients showed higher values of bad breath. Men have halitosis more often $(67 \%)$ than women $(32 \%)$.
\end{abstract}

As bad breath is caused by factors outside the mouth in only $15 \%$ of the cases, most likely, general illness cannot change this parameter.

Keywords: gastrointestinal diseases, liver diseases, bad breath, halitosis

\section{INTRODUCTION}

Halitosis is an oral health condition characterized by unpleasant odor from the oral cavity. The exact pathophysiological mechanism is unclear. It has been attributed mainly to oral pathologies. It is estimated to be found in around 50 to $60 \%$ of the general population and that carries serious personal and social repercussions $(1,2)$.

\footnotetext{
Address for correspondence:

Vladimir Panov

Faculty of Dental Medicine,

Medical University of Varna

84 Tzar Osvoboditel Blvd.

9002 Varna, Bulgaria

e-mail:vl_panov@abv.bg
}

Received: September 10, 2016

Accepted: December 12, 2016
Oral causes are related to deep carious lesions, periodontal disease, oral infections, peri-implant disease, pericoronitis, mucosal ulcerations, impacted food or debris, tongue coating, certain foods, poor oral health care, improper cleaning of dentures, dry mouth, tobacco products, and other medical conditions (3).

Bad breath can be a sign of other health problems in about $15 \%$ of the cases. Halitosis resulting from gastrointestinal disorders is considered to be extremely rare. However, halitosis has often been reported among the symptoms related to Helicobacter pylori infection and gastroesophageal reflux disease $(4,5,6)$. Dentists should pay more attention to $H$. $p y$ lori infection and refer these patients to internists to prevent further gastrointestinal complications and probable malignancies $(7,8)$. 
The results of a study show that patients with ulcerative colitis had more oral signs and symptoms, including halitosis, than the patients in the normal group. Further researches on oral lesions in ulcerative colitis are also recommended so that these diseases can be better understood $(9,10)$. Gastrointestinal and endocrine disorders may contribute to the problem $(11,12)$. Data suggest that halitosis is not typically associated with erosive gastroesophageal reflux disease and the presence of esophageal mucosal damage (13). High values of volatile sulfur compounds are not associated with the presence of hepatic disease $(14,15)$.

Few studies have been performed in order to use breath analysis as a screening tool for liver disease diagnosis. Sulur-containing compounds, such as mercaptans, dimethyl sulfide, and hydrogen sulfide are proposed as liver cancer biomarkers. Little information is available about the possible use of VOCs in patients with liver cirrhosis (17).

In colorectal cancer, hydrogen sulfide has been identified in breath as a product of gastrointestinal bacterial fermentation (17). Sulfur-containing compounds, such as dimethyl sulfide, hydrogen sulfide and mercaptans are proposed as liver cancer biomarkers (1).

Breath is a medium for the analysis of VOCs, as they are generated within the body via the blood and appear in exhaled breath. Their concentration is a parts-per-million by volume (ppmv) and parts-perbillion by volume (ppbv) levels or lower. The trace compounds produced in the oral cavity do not necessarily enter the blood stream, but they do appear on exhaled breath. Analysis of the concentrations of VOCs in breath can provide an indicator of the metabolic status, allowing a distinction between healthy and diseased states. Irritating concentration is reported as $10 \mathrm{ppm}$, and olfactory fatigue and nerve paralysis are reported at 100 and 150 ppm (17).

\section{AIM}

The aim of this paper is to investigate the presence and degree of halitosis in gastrointestinal and liver diseases.

\section{MATERIALS AND METHODS}

We examined 55 patients with various hepatic and gastrointestinal diseases. The participants were
42 patients with different liver pathology (chronic hepatitis B and C, liver cirrhosis, alcoholic and autoimmune hepatitis, Wilson's disease) and 13 patients with gastrointestinal diseases (colon polyps, Crohn's disease, achalasia, MALT lymphoma, colitis, diarrhea, colonic diverticulosis, peritoneal carcinomatosis and a condition after resection of gastric carcinoma), with a mean age of 55.16 (range 20-78, $\mathrm{SD} \pm 14.97)$ years. The study group included 18 women (32.7\%; range 28-77 years) and 37 men (67.3\%; range 20-78 years). Patients were examined at the Department of Gastroenterology at the Department of Internal Medicine at University Hospital "St. Ivan Rilsky” Medical Faculty, Medical University, Sofia, Bulgaria.

We compared the results with those of 79 patients with good general health at an average age of 45.7 (range 3-82). The men included in this study were at an average age 42.1, and the average age of women was 46.2 years (control group).

With an apparatus used to measure bad breath we investigated the levels of odors in patients. We used HC-212SF FitScan Breath Checker of Tanita Corporation of America, Inc. The apparatus quantifies volatile sulfur compounds, hydrogen sulfide (H2S), mercaptan $(\mathrm{CH} 3 \mathrm{SH})$, dimethyl sulfide (CH3$\mathrm{S}-\mathrm{CH} 3$ ) and hydrocarbon from the mouth on a scale from 0 to 5. It works with a semiconductor gas sensor. The nature of the odors is classified into six grades 0 : no odor, 1 : barely noticeable, 2 : slight but clearly noticeable, 3 : moderate, 4 : strong and 5 : very strong.

When the apparatus is switched on we must wait for about five seconds for it to calibrate. The time is counted on the display from 5 to 0 . As soon as the unit is ready for testing, it beeps. The patient should blow from a distance of about one centimeter. If the patient does not blow out immediately after the beeper or does not hit the sensor, the unit displays error. This reduces the possibility of errors. After 500 samples, including errors, the unit stops working because it believes that the integrity of the data will already be high enough.

\section{RESULTS AND DISCUSSION}

In the first group in women we found the average value of bad breath to be 0.41 with the average age being 56.9 years (median 56.89). 


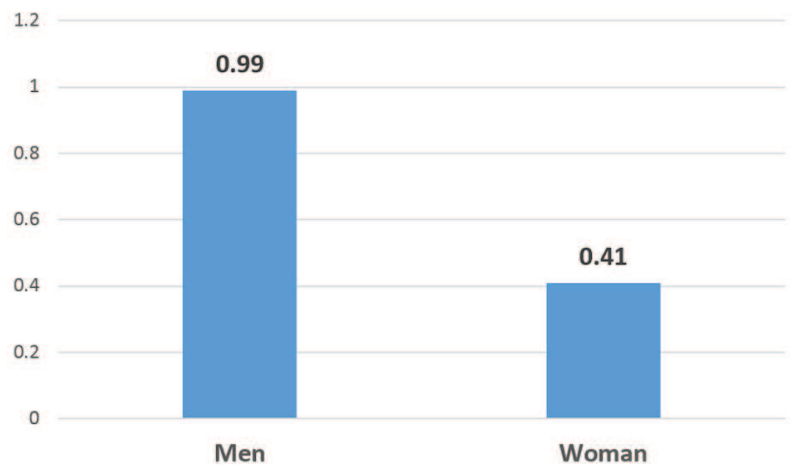

Fig 1. Bad breath in gastrointestinal and liver diseases-patients according to sex

In men aged 52.81 years (median 55) we recorded an average value of 0.99 (Fig. 1).

These studies indicate that levels of bad breath were significantly higher in men - more than two times, which is in contrast to the group of patients without systemic diseases ( 0.92 in men and 1.02 in women)(16). The results for men in both groups were similar ( 0.99 vs. 0.92$)$. We found a large difference in women where levels in those with disease are much lower compared to the control group (0.41 vs. 1.02). These results indicate that women with gastrointestinal and liver diseases have the lowest values from all tested groups.

Ciçek Y et al. found in 2010 that halitosis was statistically higher in men compared to women and there were statistically significant variations between women and men in the right-handed participant group (18).

Table 1. Degrees of bad breath in gastrointestinal and liver diseases

\begin{tabular}{|l|c|c|c|c|}
\hline Value & $0 / \%$ & $1 / \%$ & $2 / \%$ & $3 / \%$ \\
\hline men & $12 / 33.3 \%$ & $15 / 41.7 \%$ & $6 / 16.7 \%$ & $3 / 8.3 \%$ \\
\hline women & $13 / 68.4 \%$ & $5 / 26.3 \%$ & $0 / 0 \%$ & $1 / 5.3 \%$ \\
\hline total & $25 / 45,4 \%$ & $20 / 36,4 \%$ & $6 / 10.9 \%$ & $4 / 7.3 \%$ \\
\hline
\end{tabular}

The distribution of degrees of halitosis in the gastrointestinal and liver disease patients shows they rarely have the value 3 , both men and women. In men, the most common value is 1 (in $42 \%$ ), while in women most cases have a value of 0 (in 68\%) or no bad smell from the mouth. We did not observe values 4 and 5 (Tabl. 1).

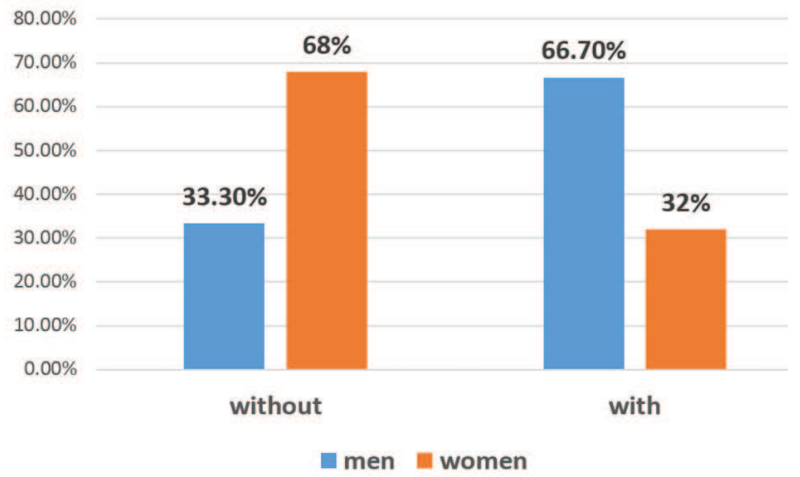

Fig. 2. Distribution of patients with and without a bad smell from the mouth

The gender breakdown shows that men without bad breath are two times fewer than women without halitosis (Fig. 2).

We can see that nearly $70 \%$ of women had no bad breath. We found lack of bad breath in men two times less frequently.

In the control group we observed that $43 \%$ of the men and 25\% of the women do not have a bad odor from the mouth. The survey found major differences in the two groups.

When allocating gastrointestinal patients into three age groups we found values in the group of 20 40 years old of 0.59 on average. 11 patients at a mean age of 29.6 years (rank 20-25) and median 30 were part of this group.

In the group of 41-60 year olds there were 23 people at an average age of 53.2 years (rank 42-60) and median 54.5, the average value was 0.89 .

In the third group of liver and gastrointestinal disease compromised patients of over 61 years, 21 people were included at an average age of 68 years (grade 61-78) and median 69. The values of bad breath that we measured averaged at 0.79 .

As previously reported, values of bad breath in the group of patients without common diseases for the 15 individuals under the age of 18 are 0.87 ( $S D \pm 1.36)$, median 0 , rank $0-5$. For the 32 patients in the 19-50 age range group, the average value is 1 (SD \pm 0.98$)$, median 1, rank $0-3$. For the group of 32 patients over 51 years of age the average value is 1.5 ( $\mathrm{SD} \pm 0.95$ ), median 2, rank 0-3 (16).

In both groups we observed an augmentation of the values of bad breath with the increase of age. 
In 2007 in Brazil the prevalence of persistent oral malodor was $15 \%$. Halitosis was about three times higher in men than in females, regardless of age. The results show more than three times higher incidence of unpleasant oral odor in people over 20 years of age compared with those aged 20 years or under (19). Our study confirms the findings of Ciçek $Y$ et al. and Nadanovsky $P$ et al.

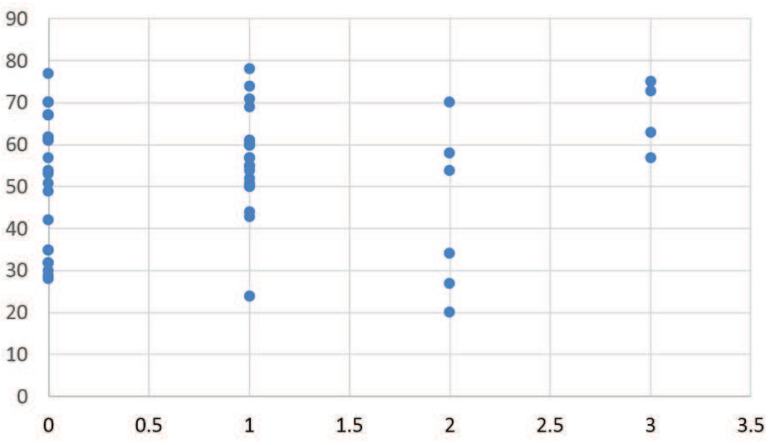

Fig. 3. Distribution of the degree of halitosis in relation to age in years in gastrointestinal diseases

From the distribution of the degree of halitosis in relation to age in years in gastrointestinal diseases (Fig. 3) it can be seen that we have registered the highest value -3 in older patients, which is a confirmation of the fact that the values of bad breath are greater in older people.

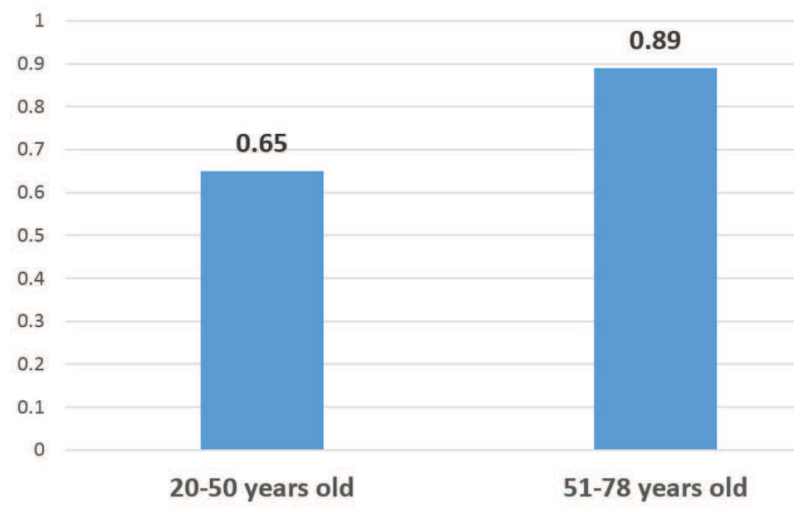

Fig. 4. Average degree of halitosis in gastrointestinal patients according to age

We divided patients with gastrointestinal diseases into two groups according to age. We saw an average of bad breath 0.65 in younger (20-50 years) and higher -0.89 in older (51-78 years) patients (Fig.
4). An increase of halitosis is clearly seen with the increase of age. We received similar results also in the studied group without common diseases.

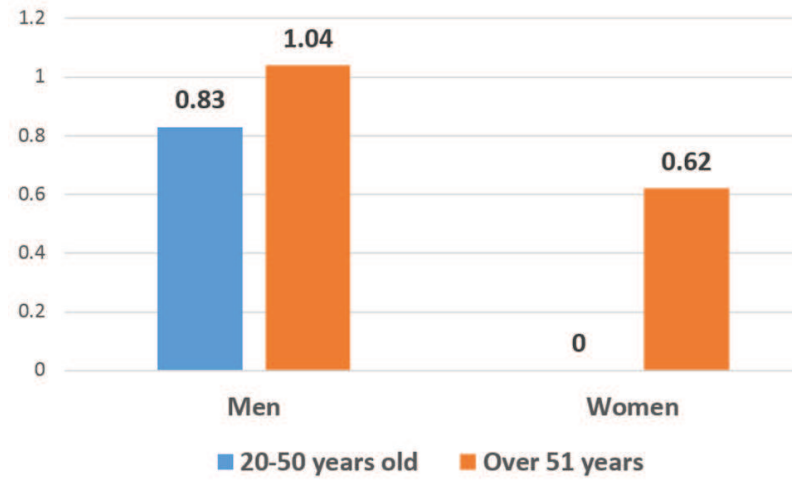

Fig. 5. Average values of bad breath in gastrointestinal diseases patients according to age and gender

We measured higher levels of bad breath in men both in the group of younger people (20-50 years) and in the older group, with values of 0.83 and 1.04 , respectively. In women, the values were 0 in the group of younger females and 0.62 in the elderly group (over 51 years) (Fig. 5).

We observed an increase in the average values of bad breath both in men and women with increasing age. We found that young women with gastrointestinal and liver diseases do not have any bad breath, which confirmed the investigations of Guglielmi M et al. in 2014 (14).

Our study found significant differences between the genders. This is most likely due to the maintenance of good oral hygiene by the women caused by the presence of a general medical condition, especially in young women. As we know, in most cases, bad breath is caused by pathologies in the mouth. In men we observed deterioration of this parameter, probably due to lack of motivation and reduced care for oral health. The growth of bad breath with age in both sexes is most likely due to the increase of oral pathology with age. As bad breath is caused by factors outside the mouth in only $15 \%$ of the cases, most likely, general illness does not change this parameter.

\section{CONCLUSIONS}

Within the limitations of this study, high values of halitosis were not mandatorily associated with 
the presence of gastrointestinal and hepatic disease. We observed an increase of the values with age. We found higher values in males. Men have halitosis (67\%) more often in comparison with women (32\%). Our results are in contrast with some investigations $(1,3,7,14)$, but confirm the results of other studies $(6,8,11,12)$.

\section{REFERENCES}

1. Tomás Carmona I, Limeres Posse J, Diz Dios P, Fernández Feijoo J, Vázquez García E, Extraoral etiology of halitosis. Med Oral. 2001 Jan-Feb;6(1):40-47.

2. Mokeem SA. Halitosis: a review of the etiologic factors and association with systemic conditions and its management. J Contemp Dent Pract. 2014 Nov 1;15(6):806-811.

3. Cortelli JR, Barbosa MD, Westphal MA. Halitosis: a review of associated factors and therapeutic approach. Braz Oral Res. 2008;22 Suppl 1:44-54.

4. Sivan Kinberg, Miki Stein, Nataly Zion, Ron Shaoul, The gastrointestinal aspects of halitosis, Can J Gastroenterol. 2010 Sep; 24(9): 552-556.

5. Krasteva A, Panov Vl, Krasteva A, Kisselova A. Oral cavity and systemic diseases - Helicobacter pylori and dentistry. Biotechnol \& Biotechnol EQ. 2011;25:2447-541.

6. Krasteva A, A Kisselova, V Dineva, Vl Panov, A Ivanova, Z Krastev. Presence of helicobacter pylori in patients with oral malodor. J of IMAB 2013; 19(4):419-421.

7. HajiFattahi F, Hesari M, Zojaji H, Sarlati F, Relationship of Halitosis with Gastric Helicobacter Pylori Infection. J Dent (Tehran). 2015 Mar;12(3):200-205.

8. Adler I, Muiño A, Aguas S, Harada L, Diaz M, Lence A, Labbrozzi M1, Muiño JM, Elsner B, Avagnina A, Denninghoff V, Helicobacter pylori and oral pathology: relationship with the gastric infection. World J Gastroenterol. 2014 Aug 7;20(29):9922-9935.

9. Elahi M, Telkabadi M, Samadi V, Vakili H, Association of oral manifestations with ulcerative colitis. Gastroenterol Hepatol Bed Bench. 2012 Summer;5(3):155-160.

10. Krasteva A, Panov Vl, Krasteva A, Kisselova A. Oral cavity and systemic diseases - inflammatory bowel diseases. Biotechnol. \& Biotechnol. Eq., 2011, 25(2), 2305-2309.
11. Bollen CM, Beikler, Halitosis: the multidisciplinary approach, T.Int J Oral Sci. 2012 Jun;4(2):55-63.

12. Krasteva A, Panov Vl, Krasteva A, Kisselova A. Oral cavity and systemic diseases - GERD. Biotechnol. \& Biotechnol. Eq., 2012, 26(2), 2823-2825.

13. Kislig $\mathrm{K}$, Wilder-Smith $\mathrm{CH}$, Bornstein MM, Lussi A, Seemann R, Halitosis and tongue coating in patients with erosive gastroesophageal reflux disease versus nonerosive gastroesophageal reflux disease. Clin Oral Investig. 2013 Jan;17(1):159-165.

14. Guglielmi M, Beushausen M, Feng C, Beech A, Baur D, Halitosis as a product of hepatic disease. SADJ. 2014 Sep;69(8):364-367.

15. Panov Vl. Oral manifestations of hepatitis $C$ virus, J of IMAB 2013, 19(4):377-379.

16. Panov Vl, Bad breath and its association with age and gender, Scripta Scientifica Medicinae Dentalis, 2 (2), 2016, 7-10.

17. Lourenço $\mathrm{C}, \mathrm{Cl}$ Turner, Breath Analysis in Disease Diagnosis: Methodological Considerations and Applications, Metabolites. 2014 Jun; 4(2): 465-498.

18. Ciçek Y, Arabaci T, Canakçi CF. Evaluation of oral malodour in left- and right-handed individuals. Laterality. 2010 May;15(3):317-326.

19. Nadanovsky P, Carvalho LB, Ponce de Leon A., Oral malodour and its association with age and sex in a general population in Brazil. Oral Dis. 2007 Jan;13(1):105-109. 